\title{
Towards Identifying Influential Indicators for Predicting Football Club Stock Price
}

\author{
Ashish Chouhan ${ }^{1 *}$, Tanmay Dhimate ${ }^{2}$, Srivathsa Saya ${ }^{3}$, Ojal Neelawarna ${ }^{4}$, \\ Tripti Pandya ${ }^{5}$, Ajinkya Prabhune ${ }^{6}$, and Manoj B. Chandak ${ }^{7}$ \\ ${ }^{1-6}$ SRH Hochschule Heidelberg, Germany \\ ${ }^{7}$ Ramdeobaba College of Engineering and Management, India
}

\section{ABSTRACT}

In this paper, we present a novel framework to identify influential indicators necessary for predicting a football club's stock price. The stock price movements are not random, but gets manipulated due to external factors. People's views and reactions on social media platforms are a few of the factors that influence the brand value of an organisation and alters the stock prices in a certain way. Hence, we aim to understand and identify the indicators that affect the stock price of Manchester United Football Club. In order to predict the stock price, the data is collected from social media, stock market, and football event for the 2018-2019 season of English Premier League (EPL). The primary challenge in accurately predicting the stock price is to identify unique features from the sources and explain how they influence the stock market. The framework is designed on the principles of microservices architecture pattern, and consists of five groups of microservices: (a) Data Extraction (b) NLP Pre-processor (c) Financial Indicator (d) Feature Identifier, and (e) Prediction Model Evaluator. For coordinating the execution of the microservices, workflows are orchestrated through the orchestrator component of the framework. For step-wise execution of the workflow, the workflow is divided into four sub-workflows. Finally, after evaluation of the framework, out of various indicators, Financial Indicators (FI) and Sentiment Indicators (SI), are identified as the prominent indicators that show the highest impact for predicting the fluctuation in the stock price of Manchester United football club.

KEY WORDS: MICROSERVICES, FEATURE SELECTION, TOPIC MODELING, STOCK PRICE PREDICTION, NATURAL LANGUAGE PROCESSING

\section{INTRODUCTION}

Prediction of a stock price is an important and complex issue in the financial sector as the organisation's current situation and future growth can be interpreted with the help of these values [Sahoo PK, 2015]. The stock prices of an organisation not only depend on the financial

\section{ARTICLE INFORMATION}

*Corresponding Author:1ashish.chouhan@srh.de

Received 17th Oct 2020 Accepted after revision 29th Dec 2020

Print ISSN: 0974-6455 Online ISSN: 2321-4007 CODEN: BBRCBA

Thomson Reuters ISI Web of Science Clarivate Analytics USA and Crossref Indexed Journal

\section{Clarivate
Analytics}

NAAS Journal Score 2020 (4.31)

A Society of Science and Nature Publication,

Bhopal India 2020. All rights reserved.

Online Contents Available at: http//www.bbrc.in/

Doi: http://dx.doi.org/10.21786/bbrc/13.14/12 status, but also on the socio-economic condition around the globe. Not only the country's economic growth has a direct impact on the stock market, but also the social events have a considerable impact on them [Laidroo L, 2008]. With the advent of social media, people are now expressing their thoughts and reactions about an organisation, its products, and related news on various social media platforms. Similarly, for a game like a football, that is globally followed, fans express their emotions on these platforms. It is safe to assume that these reactions on social media will have an impact on stock variations. Social media is no longer just a medium to connect people but has also become a medium for marketing and advertising. 
Football clubs have started to view themselves as brands and are getting influenced by the social media platforms that include Facebook, Twitter, Instagram, YouTube, and many more [Appel G, 2020]. Football fans have a relationship with their favourite team, along with a very high level of emotional connection, recognition, and engagement [Abosag I, 2012]. Thus, an aggregation of public reaction, stock price, and football events can be used to predict the stock prices. Sentiment analysis and stock price prediction using Machine Learning (ML) are studied extensively for an organisation's growth prediction and analysis [Park H, 2014]. [Hegazy 0, 2013] in their paper, propose an ML model to predict the stock price value after analyzing the historical data on inventories and technical indicators. The paper published by Dipti Sharma et al. [Sharma D, 2019] provides an overview of various techniques that are helpful when working on sentiment analysis of social media data.

Figure 1: Architecture of Framework

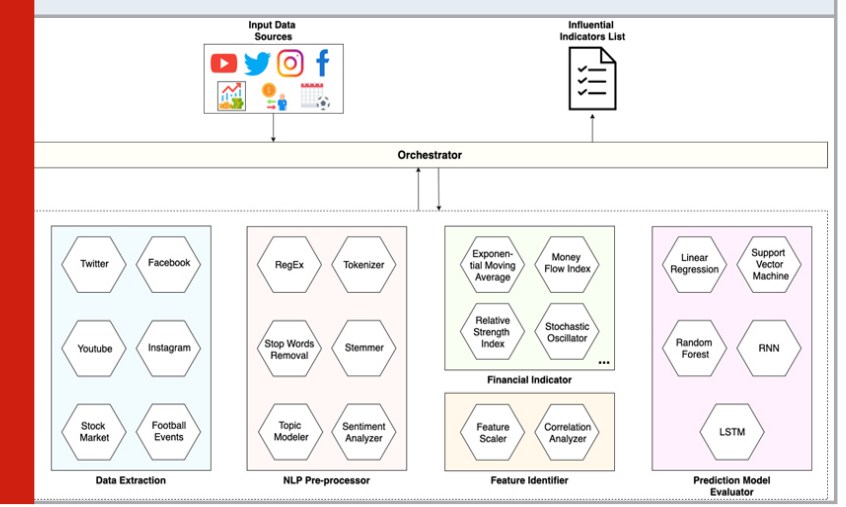

In the paper proposed by [Devi KN, 2019], insights regarding the impact of different indicators such as sentiment indicators, financial indicators, and technical indicators on stock price prediction are provided. Stock market information can distort the overall trend even due to small variation in price movements, thus creating noise in the overall prediction. This characteristic is considered as one of the challenges as the stock prices keep fluctuating [Jin Z, 2020]. Since the stock market generates large quantities of data every day, it is difficult for investment managers and stockbrokers to consider all of the present and historical information for estimating future stock patterns [Kalyani J, 2016]. Hence, this research paper aims to identify indicators that can have an impact on the fluctuation of the stock price. Following are the features of the presented framework:

- A microservice-based architecture that allows easy extensibility with the addition of new microservices, and the execution of customized workflows for microservices co-ordination.

- Systematic workflows for identifying indicators for prediction of the stock price.

- The unique set of indicators are calculated and considered in the framework that includes Financial
Indicators (FI), Sentiment Indicators (SI), Event Indicators (EI), and Topic Indicators (TI).

The remainder of this paper is structured as follows. Section 2 introduces the framework and the four subworkflows for identifying the influential indicators for predicting the Manchester United football stock price. In Section 3, we present the evaluation and discussion of the results obtained from a different combination of indicators. Finally, in Section 4, we conclude the paper and give a brief outline of our ongoing work.

\section{METHODOLOGY}

In this section, we present the framework, as shown in Figure 1. This framework is based on the microservice architecture pattern consisting of a systematically arranged group of microservices, where each microservice is responsible for a specific task. The Orchestrator component present in the framework coordinates different microservices in the form of a complex workflow to achieve the task. In the current version of the framework, the workflow is further divided into four sub-workflows that are further explained in this section. The source code of the framework is available online. 1

Sub-workflow-A for Data Extraction: This sub-workflow is responsible for extracting the EPL data from social media, stock market, and football event for the 20182019 season. The microservices responsible for extracting social media data comprises of Twitter, Facebook, YouTube, and Instagram, where data is extracted through scrapping official social media handles of Manchester United Football Club, namely Twitter2, Instagram3, Facebook4, and YouTube5. The social media data consisted of comments, timestamps, username, profile ID, and number of likes. Out of these extracted social media data, comments and timestamp were considered for further processing in the framework. A total of 8,90,725 comments are collected from social media data sources. Similarly, the Stock Market microservice extracts the financial data of Manchester United Football Club with the help of APIs from Yahoo! Finance6 comprising of an opening price, closing price, low price, high price, and volume of trade for each working day.

The Football Events microservice extracts the betting odds and match results obtained from Oddsportal7 website. This website provides betting data for Manchester United and the opposition team along with the match results. The Football Event microservice also extracts the League Standings of Manchester United for each season from the official EPL8 website. The extracted data from different microservices are further concatenated by the Orchestrator and stored in Result A, as shown in Figure 2. 
Figure 2: Sub-workflow-A for Data Extraction

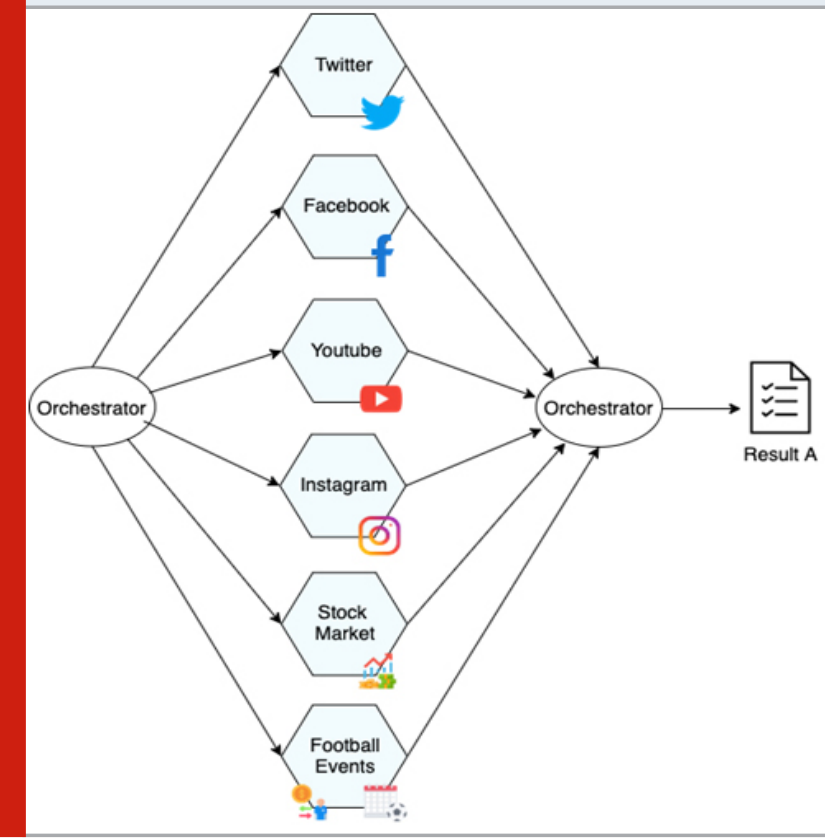

Sub-workflow-B for NLP Pre-processor: The result obtained from sub-workflow-A (Result A as shown in Figure 2) is provided to sub-workflow-B (as shown in Figure 3). In this sub-workflow, the comments collected from different social media platforms are provided to the microservices to perform data cleaning in order to generate SI comprising of sentiment score and TI consisting of the probability distribution of topics on the comments. Since the data extracted from social media comprise of HTML tags, a regular expression removes the HTML tags in RegEx microservice.

1. https://twitter.com/ManUtd

2. h t t p s : // w w w. in s t a g r a m. c o m/ manchesterunited/?hl=en

3. https://www.facebook.com/manchesterunited

4. https://www.youtube.com/channel/ UC6yW44UGJJBvYTlfC7CRg2Q

5. https://in.finance.yahoo.com/

6. https://www.oddsportal.com/

7. https://www.premierleague.com/

After the removal of HTML tags, the cleaned comments are provided to the Tokenizer microservice to separate the comments into a token of words using the NLTK library. As stop-words do not have any impact on the sentiment score,

the tokens obtained from Tokenizer microservice is provided to the Stop Words Removal microservice, where stop-words are removed using a stop-words dictionary present in NLTK library. For example, words like is, are, that, the are some of the stop-words that are removed by this microservice. To calculate the precise sentiment score, the tokens must be in their root form. Therefore, the
Stemmer microservice is executed to convert the words into their root form. For example, the word 'laziness', when stemmed gives 'lazy'. The tokens obtained from the Stemmer microservice are provided concurrently to two microservices, namely, Sentiment Analyzer, and Topic Modeler. The Sentiment Analyzer microservice focuses on obtaining the sentiment scores, and the Topic Modeler microservice realized using the Non-negative Matrix Factorization (NMF) method provides probabilistic distribution in four important topics that are relevant to the group of comments. The identified four topics include Match Result, Management, Player Transfers, and Birthdays events for the club players. Result A obtained from sub-workflow A gets updated with the SI and TI, and an updated result is obtained (Result A' as shown in Figure 3).

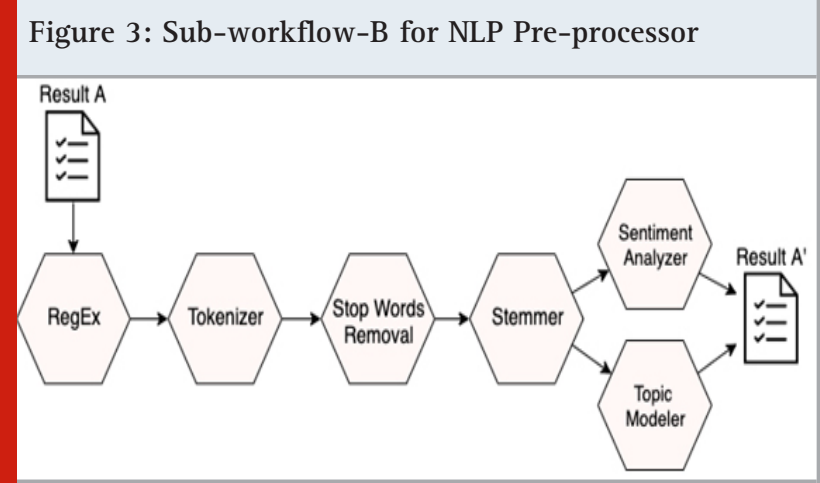

Sub-workflow-C for Feature Identifier: In this subworkflow, the FIs are calculated using the financial data present in Result A'. The input, Result A' as shown in Figure 4, is processed by the Orchestrator to calculate five FIs. The five FIs are determined using the equations stated below.

- Exponential Moving Average (EMA): Using Eq. 1, EMA is a FI that calculates the exponential moving average of a field over a given period [Wan X, 2019].

$\mathrm{EMA}=[\alpha *$ TClose $]+[(1-\alpha) *$ YEMA $]$

where TClose is Today's Close value, YEMA is Yesterday's EMA value, and $\alpha$, a constant smoothing factor between 0 and 1 , represents the degree of weighing decrease.

- Money Flow Index (MFI): MFI FI as calculated in Eq.4, measures the strength of money in and out of security [Wan X, 2019].

Money Flow $(\mathrm{MF})=$ Typical Price $*$ Volume

where Typical Price is given by, Typical Price $=($ High + Low + Close) $/ 3$ and Volume is the amount of trade performed, i.e., selling and buying of stocks. 
Money Ratio $(\mathrm{MR})=($ Positive MF /Negative MF $)$

(3)

If today's Typical Price is greater than yesterday's Typical Price, it is considered Positive Money Flow. If today's Typical Price is less than yesterday's Typical Price, it is considered Negative Money Flow. The Positive Money Flow is the sum of the Positive Money over a specific number of periods, and the Negative Money Flow is the sum of the Negative Money over a specific number of periods.

$\mathrm{MFI}=100-[100 /(1+\mathrm{MR})]$

- Relative Force Index (RSI): RSI FI is calculated using Eq. 5 and is a strong momentum indicator that compares the extent of recent losses to that of recent gains to assess the overbought and oversold conditions of an asset [Wan X, 2019].

$\mathrm{RSI}=100-[100 /(1+\mathrm{RS})]$

where RS = Average of Upward Price Change/Average of Downward Price Change.

- Stochastic 0scillator (\%K ): \%K FI is defined as the measure of the difference between the Current Closing price value of a security and its Lowest Low price, relative to the difference between its Highest High price and its Lowest Low price for a given period as shown in Eq. 6 [Wan X, 2019].

$\% \mathrm{~K}=$ (Current Close - Lowest Low) / (Highest High Lowest Low) $* 100$

- Moving Average Convergence/Divergence (MACD): MACD FI calculates the difference between a short and a long-term moving average for a given field. The equation for calculating MACD and its signal is as calculated in Eq. 7 and Eq. 8 [Wan X, 2019].

MACD $=[0.075 *$ EMA of Closing prices $]-[0.15 *$ EMA of Closing prices]

\section{Signal Line $=0.2 *$ EMA of MACD}

After calculating the five FIs, out of these five FIs, the indicators having a strong correlation with the closing stock value of next working day are selected. The Correlation Analyzer microservice carries out the task to evaluate the strength of the relationship between each of these five FIs with the closing stock value of next working day. Based on the correlation score, three out of the five FIs were finalised. These three FIs include EMA, RSI, and MFI. These selected FI, along with SI, TI, and EI, are provided to Feature Scaler microservice. The reason to perform feature scaling is to scale values present in different indicators as a different range of values would affect the efficiency of the model [Kalyani $\mathrm{J}, 2016]$. Therefore, feature scaling is performed to scale the values in the same range for all the indicators by Feature Scaler microservice and Result B as shown in Figure 4 is obtained.

Figure 4: Sub-workflow-C for Feature Identification

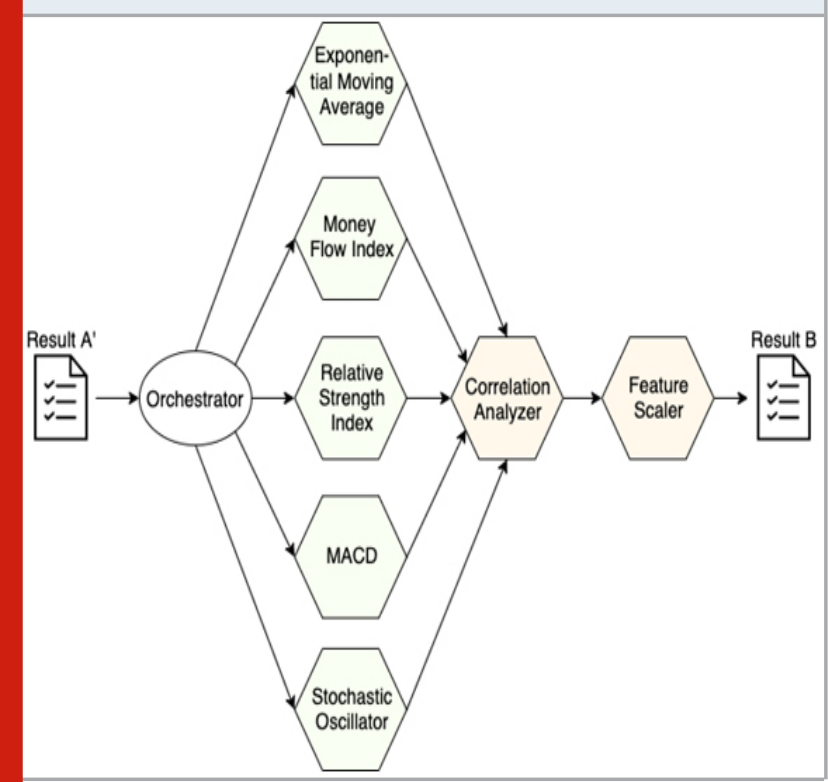

Sub-workflow-D for Model Evaluations: This subworkflow aims to optimise the identification of the indicators by evaluating the framework against five prediction models. These five prediction models include Linear Regression (LR), Random Forest Regression (RF), Support Vector Regression (SVR), Long Short Term Memory (LSTM), and Recurrent Neural Network (RNN). The models are trained with a different combination of indicators to predict the dependent variable, i.e., closing stock value of next working day. As shown in Figure 5, Result B obtained from sub-workflow-C is provided as an input to the Orchestrator. The Orchestrator gives different combinations of FI, SI, TI, and EI to all the five models and obtains RMSE (Root Mean Square Error) score as an output from each model. The obtained RMSE scores are stored in a matrix of $\mathrm{m} \times \mathrm{n}$ (Result $\mathrm{C}$ as shown in Figure 5) where different combinations of indicators are present in the row, and prediction models are present in the columns with RMSE scores present in the intersection cells of the rows and columns.

\section{EVALUATION AND DISCUSSION}

Table 1 provides the results obtained by the framework on a different combination of indicators for prediction models. For the evaluation of models, RMSE (Root Mean Square Error) evaluation technique is used [Willmott CJ, 2005]. In Table 1, the columns LR, RF, SVR, RNN, and LSTM refer to the Prediction models, namely, Linear Regression, Random Forest Regression, Support Vector 
Regression, Recurrent Neural Network, and Long Short Term Memory respectively. Similarly, FI indicates the financial indicators (EMA, MFI, RSI), and SI indicates the sentiment indicators (sentiment scores). Event indicators and topic indicators are represented as EI and TI respectively, followed by CI that represents the combination of all four indicators, i.e., FI, SI, TI, and EI. Various combinations of these indicators were provided to the models, and the evaluation results

Figure 5: Sub-workflow-D for Model Evaluation

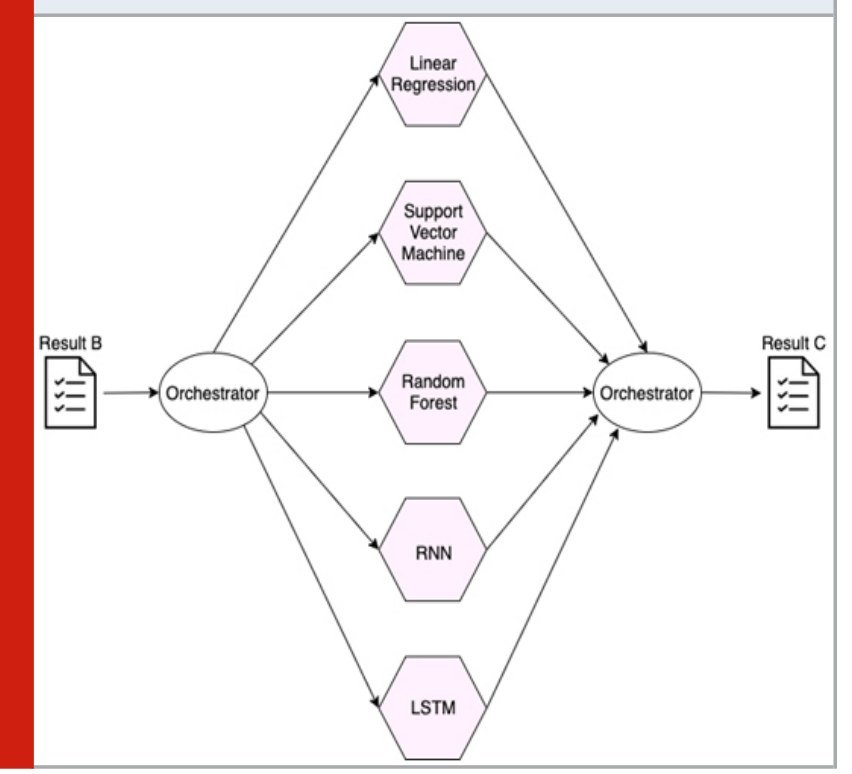

Table 1. RMSE scores for Prediction models

\begin{tabular}{|c|c|c|c|c|c|}
\hline Indicators & LR & RF & SVR & RNN & LSTM \\
\hline SI & 3.2015 & 0.4453 & 2.6540 & 0.0496 & $\mathbf{0 . 0 1 2 1}$ \\
FI & 0.1429 & 0.0158 & 0.1084 & 0.3117 & 0.3117 \\
TI & 3.1442 & 0.4640 & 2.3138 & 0.0216 & 0.0164 \\
EI & 3.3765 & 2.1485 & 3.6382 & 0.1925 & 0.1925 \\
FI + SI & 0.1429 & 0.0174 & 0.1153 & 0.3117 & 0.3117 \\
EI + SI & 3.2578 & 0.2961 & 3.4889 & 0.1925 & 0.0139 \\
EI + FI & 0.1443 & $\mathbf{0 . 0 1 3 6}$ & 0.1386 & 0.3117 & 0.1015 \\
TI + FI & 0.1441 & 0.0203 & 0.1214 & 0.3089 & 0.3117 \\
TI + EI & 3.1743 & 0.3391 & 3.1235 & 0.0216 & 0.0216 \\
TI + SI & 3.3953 & 0.4814 & 2.3259 & 0.0216 & 0.0175 \\
FI + SI + EI & 0.1439 & 0.0151 & 0.1338 & 0.1141 & 0.1118 \\
SI + EI + TI & 3.4553 & 0.3801 & 3.1020 & 0.0216 & 0.0216 \\
FI+ SI + TI & 0.1457 & 0.0191 & 0.1224 & 0.3117 & 0.3117 \\
FI + EI + TI & 0.1452 & 0.0190 & 0.1323 & 0.3117 & 0.0961 \\
CI & 0.1473 & $\mathbf{0 . 0 1 6 8}$ & 0.1310 & 0.3117 & 0.1064 \\
\hline
\end{tabular}

were obtained. From Table 1, it can be observed that the RMSE scores are lower for most of the indicators combination for the RF and LSTM models. However, our focus is on identifying the indicators that have an impact on predicting the Manchester United Football Club stock price. When FI, SI, TI, and EI are evaluated individually, then SI performs better with LSTM model having the RMSE score of 0.0121. For evaluation of a combination of indicators, a combination of FI and EI performed better for RF model with the RMSE score of 0.0136. For CI, i.e., a combination of all indicators, $\mathrm{RF}$ is the best performing model with the RMSE score of 0.0168 .

\section{CONCLUSION}

In this paper, we presented a framework to identify the indicators influencing the stock prices of Manchester United Football Club. The framework is designed on the microservices architecture pattern for allowing easy extensibility when adding or updating the framework with new features. The functionality of the framework is split into different groups of microservices. The presented version of the framework has five groups of microservices: (a) Data Extraction, (b) NLP Preprocessor, (c) Financial Indicator, (d) Feature Identifier, and (e) Prediction Model Evaluator. To handle these microservices, the framework provides an Orchestrator component. The Orchestrator allows users to describe workflows, register microservices, and coordinate their execution.

The framework is designed to calculate the FI, SI, TI, and EI from social media, stock market, and football event for the 2018-2019 season of English Premier League (EPL). Various combination of indicators are evaluated against prediction models using the RMSE scores. For the different combination of indicators, from the RMSE score, LSTM and RF models were the most efficient models. The framework finds out that the deep learning model LSTM has given an RMSE score of 0.0121 for SI followed by a combination of FI and EI with RF giving the RMSE score of 0.0136. Considering all the indicators, i.e., FI, SI, TI, and EI, RF prediction model is performing better with RMSE score of 0.0168. In our ongoing work, we are working to extend the indicators by considering the news articles, attendance of the people for matches, and sponsorship details.

\section{ACKNOWLEDGEMENTS}

We would like to thank the Department of Information, Media, and Design of SRH Heidelberg University and Department of Computer Science of Shri Ramdeobaba College of Engineering and Management (RCOEM) for supporting us during this research.

\section{REFERENCES}

Abosag I, Roper S, Hind D. Examining the relationship between brand emotion and brand extension among supporters of professional football clubs. European Journal of marketing, Vol:46(9), 2012, pp:1233-1251.

Appel G, Grewal L, Hadi R, Stephen AT. The future of social media in marketing. Journal of the Academy of Marketing Science, Vol:48(1), 2020, pp:79-95.

Devi KN, Krishnamoorthy N, Aparna KS. Social Media Aided Sentiment Analysis for Stock Prediction. International Journal of Innovative Technology and 
Exploring Engineering, Vol:9(2), 2019, pp:112-116. Hegazy 0, Soliman OS, Salam MA. A machine learning model for stock market prediction. International Journal of Computer Science and Telecommunications, Vol:4(12), 2013, pp:1402-7351.

Jin Z, Yang Y, Liu Y. Stock closing price prediction based on sentiment analysis and LSTM. Neural Computing and Applications, Vol:32, 2020, pp:9713-9729.

Kalyani J, Bharathi P, Jyothi P. "Stock trend prediction using news sentiment analysis," arXiv preprint arXiv:1607. 01958, 2016.

Laidroo L. Public announcement induced market reaction on Baltic stock exchanges. Baltic Journal of Management, Vol:3(2), 2008, pp:174-192.

Park H and Kim YK. The role of social network websites in the consumer-brand relationship. Journal of Retailing and Consumer Services, Vol:21(4), 2014, pp:460-467. Sahoo PK and Charlapally K. Stock price prediction using regression analysis. International Journal of Scientific and Engineering Research, Vol:6(3), 2015, pp:1655-1659.

Sharma D, Sabharwal M, Goyal V, Vij M. "Sentiment Analysis Techniques for Social Media Data: A Review," in International Conference on Sustainable Technologies for Computational Intelligence. 1st Conference, 2019, pp:75-90.

Wan X. Influence of feature scaling on convergence of gradient iterative algorithm. Journal of Physics, Vol:1213(3), 2019, pp:1-5.

Willmott CJ and Matsuura K. Advantages of the mean absolute error (MAE) over the root mean square error (RMSE) in assessing average model performance. Climate research, Vol:30(1), 2005, pp:79-82. 